\title{
Treinamento dos Músculos do Assoalho Pélvico Associado à Gameterapia para Tratamento da Incontinência Urinária de Esforço
}

\author{
Training of Pelvic Floor Muscles and Game Therapy for Treatment \\ of Effort-related Urinary Incontinence
}

\section{RESUMO}

Objetivo: Avaliar a eficácia do TMAP associado à gameterapia em mulheres com IUE, tendo como variáveis a gravidade, a função dos MAP e o impacto sobre a vida diária. Metodologia: A pesquisa teve aprovação do Comitê de Ética e foi realizada com 10 mulheres incontinentes, de 40 a 77 anos, no período de agosto a outubro de 2017, na Clínica Escola de Fisioterapia da Universidade da Amazônia. Utilizou-se uma ficha de avaliação fisioterapêutica uroginecológica, a escala de impacto sobre a vida diária e como instrumentos de intervenção foram utilizados o Xbox Kinect $360^{\circ}$ por 12 sessões. A análise estatística foi realizada por meio do programa GraphPad Prism 5, tendo sido utilizado o teste de Wilcoxon, sendo adotado um nível alfa $5 \%(p \leq 0,05)$ como significância estatística. Resultados: Observou-se que na avaliação da perda ao esforço, ocorreu melhora no pós-tratamento $(p=0,01)$, quanto à severidade e a progressão da perda, a maioria mencionou não existir perda pós-tratamento, porém, sem significância estatística. Na palpação dinâmica, no período pré-tratamento apenas $20 \%$ (2/10) das mulheres possuíam força completa, no entanto, na reavaliação essa variável subiu para $80 \%(8 / 10)$, sendo estatisticamente significante $(p=0,01)$, bem como, no endurece $(p=0,01)$, na potência $(p=0,007)$ e no relaxamento pós-contração $(p=0,02)$. Na avaliação pós-aplicação do protocolo, $70 \%$ (7/10) das mulheres afirmaram que a incontinência urinaria não mais impactava sobre a vida diária $(p=0,007)$. Conclusão: $O$ tratamento proposto no estudo mostrou-se eficaz para a amostra em questão, na recuperação da continência urinária.

\section{DESCRITORES}

Incontinência Urinaria. Fisioterapia. Mulheres. Assoalho Pélvico.

\begin{abstract}
Objective: To evaluate the efficacy of the training of pelvic floor muscles associated with game therapy in the treatment of women with effort-related urinary incontinence. Methodology: The research was approved by the Ethics Committee and was performed with 10 incontinent women, from 40 to 77 years, from August to October 2017, at the Physiotherapy School Clinic at the University of Amazônia. An urogynecological physical therapy assessment scale was used, and the intervention tools used were the Xbox Kinect $360^{\circ}$ for 12 sessions. Statistical analysis was performed using the GraphPadPrism 5 program and the Wilcoxon test, with $(p \leq 0.05)$ as a statistical significance. Results: It was observed the loss assessment to the effort, there was improvement in the post-treatment $(p=0.01)$, as for the severity and progression of the loss, the majority mentioned there is no loss after treatment, but without statistical significance. On dynamic palpation in the pre-treatment phase, only $20 \%$ $(2 / 10)$ of the women had complete strength, however in the re-evaluation this item went up to $80 \%(8 / 10)$, being statistically significant $(p=0.01)$, as well as on the endurance $(p=0.01)$, potency $(p=0.007)$, and post-contraction relaxation $(p=0.02)$. In the post-protocol evaluation, $70 \%(7 / 10)$ of the women stated that urinary incontinence no longer impacted daily life $(p=0.007)$. Conclusion: the treatment proposed by the research proved to be effective for the sample in question in the recovery of urinary continence.
\end{abstract}

\section{DESCRIPTORS}

Urinary Incontinence. Physical Therapy. Women. Pelvic Floor.

Graduanda do Curso de Fisioterapia da Universidade da Amazônia (UNAMA).

${ }^{2}$ Fisioterapeuta, Doutora em Ciências da Reabilitação. Docente da Universidade da Amazônia e da Universidade do Estado do Pará (UEPA), Belém (PA), Brasil.

${ }^{3}$ Fisioterapeuta, Doutora em Ciências pelo Programa de Medicina Tropical/IOC/FIOCRUZ. Docente do Centro Universitário Metropolitano da Amazônia (UNIFAMAZ).

${ }^{4}$ Fisioterapeuta, Doutor em Doenças Tropicais. Docente da Universidade do Estado do Pará (UEPA), Belém (PA), Brasil.

${ }^{5}$ Fisioterapeuta, Doutora em Ciências da Reabilitação. Docente da Universidade do Estado do Pará (UEPA), Belém (PA), Brasil. 
A incontinência urinária de esforço (IUE) é caracterizada pela falta de capacidade da pessoa de manter a urina na bexiga durante um aumento da pressão intra-abdominal. Ocorre devido às disfunções dos órgãos e estruturas, como ligamentos, fáscias e músculos do assoalho pélvico (MAP), sendo considerado um dos mais delicados e esgotantes problemas de saúde, pois provoca aversão social, ocupacional, psicológica, física, sexual e econômica ${ }^{1,2}$.

No Brasil, a realização de estudos de prevalência de IUE é precária, mas considera-se alta a prevalência na população feminina, variando de $5-69 \%$, geralmente em função da idade, do nível cultural, da gravidade da disfunção e da baixa notificação. Desta forma, o impacto da incontinência sobre a vida diária dessas mulheres acarreta danos consideráveis, mas em nível nacional não existem muitas pesquisas indexadas relatando o efeito desses danos ${ }^{3,4}$.

AIUE pode ser prevenida ou tratada de forma conservadora pela fisioterapia pélvica, que realiza exercícios para o MAP, seguindo, atualmente, uma ordem cronológica do aprendizado motor e sequencialmente possibilitando propriocepção, coordenação motora, reforço muscular e treinamento funcional, o que reforça o mecanismo de continência e coordena a contração reflexa dos MAP no momento do esforço ${ }^{5-7}$.

Neste sentido, o treinamento dos músculos do assoalho pélvico (TMAP) pode utilizar a gameterapia, baseado em um videogame que utiliza uma câmera com sensor de movimento, que é operado somente pelo movimento do jogador, sem a necessidade de nenhum controle, para simular atividades que provoquem perda de urina, como obstáculos que devem ser transpostos com saltos, deslocamentos laterais, flexões de tronco, extensões de tronco e membros, além de facilitar o treino funcional dos MAP nesse momento ${ }^{8}$.

Considerando que a IUE pode ser consequência da falta de propriocepção e ineficiência dos MAP em se contrair nos momentos de aumento da pressão intra-abdominal este estudo teve como objetivo avaliar a eficácia do treinamento dos músculos do assoalho pélvico associado à gameterapia em mulheres com incontinência urinária de esforço, tendo como variáveis a gravidade, a função dos MAP e o impacto sobre a vida diária.

\section{METODOLOGIA}

Trata-se de um estudo quase experimental, aprovado pelo Comitê de Ética em Pesquisa com Seres Humanos da Universidade da Amazônia (UNAMA) (Parecer $n^{\circ}$. 2.153.058), seguindo todas as normas éticas para desenvolvimento da pesquisa, realizado no período de agosto a outubro de 2017, na Clínica Escola de Fisioterapia da Universidade da Amazônia, tendo como público alvo mulheres com IUE.

A amostragem foi por conveniência, em que panfletos contendo o objetivo, os critérios de elegibilidade, o local e o horário, foram entregues a médicos que encaminhavam as mulheres que se interessavam pelo estudo. Foram adotados como critérios de inclusão mulheres com IUE ou incontinência urinária mista (IUM), com idade de 40 a 77 anos. Foram excluídas mulheres que apresentassem problemas neurológicos, problemas cardiovasculares e que tivessem dificuldades ortopédicas para realizar a gameterapia. A amostra foi constituída por 10 mulheres. 
Como instrumento de avaliação foi usado uma ficha de avaliação fisioterapêutica dividida em anamnese e exame físico. $\mathrm{Na}$ anamnese foram colhidos dados contendo a identificação, informações da história da doença, situação da perda ao esforço (tossindo, espirrando, erguendo peso, exercitando), severidade e progressão da perda - quantidade perdida (muito, jatinhos, gotas) e impacto sobre a vida diária, que foi avaliado em um score que variava de 1 (um) ponto (nenhum impacto) a 10 (dez) pontos (muito impacto) ${ }^{(9)}$, sendo classificado em cinco categorias: (0) nada, (1-3) leve, (4-6) moderado, (7-9) grave, (10) muito grave.

No exame físico foi realizada a inspeção, em que se observou a elevação uretral e o fechamento do hiato após solicitar-se a contração voluntária dos MAP. Em seguida, foi realizada a palpação para avaliar os reflexos de estiramento dos MAP, o reflexo cutâneo anal e o reflexo clitoridiano. Ainda na palpação verificaram-se dados como a força dos MAP, a endurance (resistência dada em segundos), a potência (número de vezes que consegue contrair rapidamente) e a capacidade de relaxamento pós-contração (completo, parcial, incompleto, ausente ou retardado).

As participantes encaminhadas eram conduzidas a uma sala reservada, onde era assinado o termo de consentimento livre e esclarecido, em seguida, eram avaliadas com os procedimentos da fisioterapia pélvica: história clínica e avaliação funcional do assoalho pélvico. Na avaliação também era explicada a anátomofisiologia do assoalho pélvico. Foi aplicada também uma escala numérica de variação de 1 a 10 , para verificar o quanto a incontinência urinária (IU) impactava nas ativi- dades de vida diária, sendo que 1 significava nenhum impacto e 10 muito impacto.

Como instrumento de intervenção foi utilizado o Xbox Kinect $360^{\circ}$ (XK), que é um software que proporciona a realidade virtual dentro de seus jogos, favorecendo uma maior motivação para realização do tratamento, faixa elástica, disco proprioceptivo e halteres e o aparelho Dualpex 961 da marca Quark $®$ para eletroestimulação.

Foram realizadas 12 sessões, 2 vezes por semana, distribuídas da seguinte forma: duas sessões para percepção dos MAP, feita com propriocepção manual por 10 minutos e eletroestimulação $(50 \mathrm{~Hz}, 400$ microssegundos) por 15 minutos; duas sessões para treino de coordenação (realizar contração e relaxamento ao comando e de forma seletiva), por 20 minutos; duas sessões para treino de endurance, potência, força (utilizando o toque bidigital) e da manobra de Knack (solicitação de contração dos MAP previa e mantida a uma situação de aumento da pressão intra-abdominal, proporcionada por vários exercícios com a faixa elástica, disco proprioceptivo e halteres), totalizando 50 minutos; e quatro sessões com o XK $360^{\circ}$, por 30 minutos. As participantes foram reavaliadas sete dias depois da última sessão.

Os dados foram analisados com o Programa Microsoft Excel® (Microsoft Corporation, Redmond, WA, USA) para a tabulação dos dados, bem como para a confecção das tabelas e a análise estatística foi realizada por meio do Programa GraphPad Prism 5 (GraphPad Software Inc., San Diego, CA, USA). As variáveis foram apresentadas como a distribuição de frequências, medidas de dispersão e de tendência central. Utilizou-se o teste de 
Wilcoxon para investigar as variáveis categóricas antes e após a intervenção terapêutica, sendo adotado um nível $\alpha 5 \%(p \leq 0,05)$ como significância estatística.

\section{RESULTADOS}

Participaram da pesquisa 10 mulheres com IUE ou IUM, com média de idade de $61,4 \pm 11,14$. Observou-se que na avaliação da perda ao esforço, $60 \%$ das mulheres perdiam urina ao tossir no pré-tratamento e ocorreu melhora no pós-tratamento, em que $70 \%$ relataram não perder mais urina, com significância estatística $(p=0,01)$. Quanto à severidade e a progressão da perda, a maioria das mulheres no pré-tratamento, $30 \%$ e $50 \%$ relataram perder muita urina, em forma de jatinhos, respectivamente, posteriormente, $60 \%$ mencionaram não existir mais perda (Tabela 1).

A avaliação da pressão de contração dos MAP no período pré-tratamento apenas $20 \%$ das mulheres possuíam MAP forte e na reavaliação passou para $80 \%$, sendo estatisticamente significante $(p=0,01)$. Em relação ao endurance, os valores encontravam-se dispersos, porém obteve-se uma melhora de $60 \%$ pós-terapia $(p=0,01)$. No tópico potência, avaliou-se que $10 \%$ das mulheres eram capazes de contrair $\geq 15$ vezes, durante a reavaliação observou-se que $60 \%$ adquiriram a capacidade da contração $(p=0,007)$. Apresentavam retardo no relaxamento pós-contração $40 \%$ das mulheres, na reavaliação foi visto que $70 \%$ apresentaram melhora $(p=0,02)$ (Tabela 2).

Os dados apontam que a IUE interferiu de forma relevante no impacto sobre as atividades de vida diária. No pré-tratamento, o impacto para a vida diária encontrava-se na categoria grave em $50 \%$ das mulheres seguida da categoria muito grave (30\%), todavia, no pós-tratamento, $70 \%$ relataram não impactar

Tabela 1. Dados da situação e severidade da perda de urina no pré e pós-tratamento

\begin{tabular}{|c|c|c|c|}
\hline Variáveis & $\begin{array}{c}\text { Pré-Tratamento } \\
\mathrm{n}(\%)\end{array}$ & $\begin{array}{c}\text { Pós-Tratamento } \\
\mathrm{n}(\%)\end{array}$ & $\mathrm{p}$-valor \\
\hline Situação da Perda & & & $0,01^{*}$ \\
\hline Tossindo & $06(60)$ & $03(30)$ & \\
\hline Espirrando & $02(20)$ & - & \\
\hline Erguendo Peso & - & - & \\
\hline Exercitando & $02(20)$ & - & \\
\hline Nenhum & - & $07(70)$ & \\
\hline Severidade da perda de urina & & & 0,19 \\
\hline Muita & $03(30)$ & - & \\
\hline Jatinhos & $05(50)$ & - & \\
\hline Gotas & $02(20)$ & $04(40)$ & \\
\hline Nenhum & - & $06(60)$ & \\
\hline
\end{tabular}


Tabela 2. Dados de força, endurance, potência e relaxamento pós-contração pré e pós-tratamento

\begin{tabular}{|c|c|c|c|}
\hline Variáveis & $\begin{array}{c}\text { Pré-Tratamento } \\
\mathrm{n}(\%)\end{array}$ & $\begin{array}{c}\text { Pós-Tratamento } \\
\mathrm{n}(\%)\end{array}$ & $p$-valor \\
\hline Palpação Dinâmica (Contração Máxima) & & & $0,01^{*}$ \\
\hline Forte & $02(20)$ & $08(80)$ & \\
\hline Normal & $05(50)$ & $02(20)$ & \\
\hline Fraca & $03(30)$ & - & \\
\hline \multicolumn{4}{|l|}{ Ausente } \\
\hline Endurance & & & $0,01^{*}$ \\
\hline$\geq 10 \mathrm{seg}$ & $03(30 \%)$ & $06(60 \%)$ & \\
\hline 9 a 7 seg & $03(30 \%)$ & $02(20 \%)$ & \\
\hline 6 a 4 seg & $03(30 \%)$ & $01(10 \%)$ & \\
\hline 3 a $1 \mathrm{seg}$ & $01(10 \%)$ & $01(10 \%)$ & \\
\hline \multicolumn{4}{|l|}{ Zero } \\
\hline Potência & & & $0,007^{*}$ \\
\hline$\geq 15$ vezes & $01(10 \%)$ & $06(60 \%)$ & \\
\hline 11 a 14 vezes & $03(30 \%)$ & $03(30 \%)$ & \\
\hline 6 a 10 vezes & $03(30 \%)$ & $01(10 \%)$ & \\
\hline 1 a 5 vezes & $02(20 \%)$ & - & \\
\hline Nenhuma & $01(10 \%)$ & - & \\
\hline Relaxamento Pós-Contração & & & $0,02^{*}$ \\
\hline Completo & $04(40 \%)$ & $07(70 \%)$ & \\
\hline Parcial & $04(40 \%)$ & $01(10 \%)$ & \\
\hline Incompleto & $02(20 \%)$ & $02(20 \%)$ & \\
\hline Ausente & - & - & \\
\hline Retardado & - & - & \\
\hline
\end{tabular}

(-) Dados numéricos igual a zero. *Teste de Wilcoxon

nada na vida diária, $20 \%$ mencionaram a categoria leve e apenas $10 \%$ permaneceram na categoria muito grave, com $\mathrm{p}$-valor significante de 0,007 (Tabela 3).

\section{DISCUSSÃO}

O TMAP associado à gameterapia em mulheres com IUE e IUM apresentou resultados com melhora significativa nas variáveis a situação de perda, a função dos MAP e o impacto sobre a vida diária. A severidade da
IUE melhorou, mas não de forma significativa.

As situações de perda de urina ao esforço são aquelas que ocorrem quando existe um aumento da pressão intra-abdominal. Um dos mecanismos de manutenção da continência urinária está relacionado com a contração involuntária e rápida dos MAP, imediatamente, antes do aumento da pressão intra-abdominal, a chamada pré-contração ${ }^{9}$.

O TMAP, desde 2005, é a primeira linha de tratamento para a IU, considerado o padrão ouro de tratamento para as disfunções 
Tabela 3. Dados do impacto sobre a vida diária pré e pós-tratamento

\begin{tabular}{lccc}
\hline \multicolumn{1}{c}{ Variável } & Pré-Tratamento & Pós-Tratamento & p-valor \\
\hline Impacto Sobre a Vida & & $07(70 \%)$ & $0,007^{*}$ \\
Nenhum & - & - & \\
01 & - & - \\
02 & - & $02(20 \%)$ \\
03 & - & - \\
04 & - & - \\
05 & $02(20 \%)$ & - \\
06 & - & - \\
07 & - & - \\
08 & $02(20 \%)$ & - \\
09 & $03(30 \%)$ & $1(10 \%)$ \\
10 & $03(30 \%)$ &
\end{tabular}

(-) Dados numéricos igual a zero. ${ }^{*}$ Teste de Wilcoxon

urinárias em geral. Pode reduzir o número de episódios e o volume de perda de urina, sendo a relação custo-eficácia do TMAP é promissora ${ }^{10,11}$.

Nie et al. ${ }^{12}$, em uma metanálise para avaliar os efeitos do TMAP em mulheres com $\mathrm{IU}$, concluíram que o uso regular de TMAP melhora os sintomas e a qualidade de vida de mulheres com IU. No entanto, não ocorreu um consenso sobre o tipo ideal, a duração ou a frequência do TMAP.

Outra revisão sistemática com metanálise ${ }^{13}$ concluiu que o TMAP pode ser uma terapia eficaz para reduzir a IUE em mulheres, sugerindo que as sessões sejam curtas (10-45 min) e com uma frequência de 3 e 7 dias por semana, podendo evocar as maiores mudanças em mulheres com IU.

O TMAP proposto neste estudo foi baseado no "Treinamento dos quatro Fs": Find, Feel, Force and Follow Through, que consistem, respectivamente, em 1) aprender a contrair o assoalho pélvico, 2) dominar a contração e o relaxamento, sem co-contrações de musculaturas paralelas, 3) aumentar a força, a potência, o endurance e a pré-contração automática e, por fim, 4) dar seguimento ad eternum ao tratamento ${ }^{11}$. Ressalta-se, que atualmente este treinamento foi revisto tendo sido acrescentado mais um F: Find, Feel, Force, Follow Through and Functional Training, de forma que o Force é força, potência, endurance; no Follow Through é treinada a pré-contração; e o Functional Training é dar seguimento ao treinamento nas atividades diárias ${ }^{14,15}$. Em especial, a gameterapia foi o recurso escolhido para treinar a pré-contração.

Ademais, a gameterapia vem se tornando cada vez mais utilizada por profissionais da saúde na rotina dos tratamentos que visam à reabilitação, pois exige do praticante esforço físico e um complexo de movimentos 
corporais, que é possível ser adaptado pelo sistema do aparelho e pode ser usada para o treino funcional dos MAP de forma lúdica e animadora, desafiando as participantes a contraírem os mesmos simultaneamente ao movimento proposto ${ }^{16,17}$.

Bezerra et al. ${ }^{18}$, em ensaio clinico controlado randomizado cego, realizaram um treinamento muscular do assoalho pélvico isolado e associado à terapia de jogo em dois grupos de mulheres com IUM, durante o período do climatério. Não ocorreu diferença estatisticamente significativa entre os grupos para a pressão contração dos MAP, na severidade, frequência e impacto da IUM sobre a qualidade de vida. No entanto, ambos os tratamentos se mostraram eficazes para os sintomas de IUM. A percepção de melhoria foi muito evidenciada, de acordo com o relato das mulheres.

Peixinho et al. ${ }^{20}$ realizaram, com o Nintendo Wii Fit PlusTM, 12 sessões de 50 minutos cada, três vezes por semana. Cada sessão compreendeu 20 minutos de momento educativo, com orientações das ações durante os jogos e o treinamento prévio para a contração dos músculos transverso do abdome e do assoalho pélvico, seguido de 30 minutos de intervenção com o jogo, com duas mulheres com IU. Observaram que a terapia por

\section{REFERÊNCIAS}

1. Oliveira $M$, Lattaro U. Incontinência urinária e atividade física: uma revisão de literatura. [trabalho de conclusão de curso]. Brasília: Centro Universitário de Brasília UniCEUB; 2015.

2. Guerra TEC, Rossato C, Nunes EFC, Latorre GFS. Atuação da fisioterapia no tratamento de incontinência urinaria de esforço. Femina. 2014; 42(6):251-254. exposição à realidade virtual foi efetiva para a melhora da função muscular do assoalho pélvico e da perda urinária das participantes.

Desta forma, Nascimento et al. ${ }^{19}$ realizaram uma revisão sistemática com objetivo de discutir os efeitos da terapia por exposição à realidade virtual no fortalecimento dos músculos do assoalho pélvico, concluindo que a realidade virtual melhora a ativação dos músculos do assoalho pélvico tanto em mulheres jovens como em mulheres na pós-menopausa; sendo um recurso efetivo, seguro e com viabilidade clínica e econômica.

Como limitações deste estudo citamos o $\mathrm{n}$ amostral pequeno, o fato de não terem sido coletados dados obstétricos das participantes e ter sido grupo único. Contudo, o tratamento proposto pela pesquisa mostrou-se eficaz para a amostra em questão na recuperação da continência urinária, inferindo-se que a melhora observada pode estar relacionada à ordem cronológica de aprendizado e ao treino dos MAP proposto pelo treinamento com os fs.

\section{CONCLUSÃO}

O tratamento proposto pela pesquisa mostrou-se eficaz para a amostra em questão na recuperação da continência urinária.

3. Truroff JW, Abrams P, Andersson KE, Artibani W, Chapple $\mathrm{CR}$, Drake MJ, et al. EAU guidelines on urinary incontinence. Eur Urol. 2011; 59(3):387-400.

4. Silva Al, Almeida C, Aguiar H, Neves M, Teles MJ. Prevalência e impacto da incontinência urinária na qualidade de vida da mulher. Rev Port Med Geral Fam. 2013 29(6):364-376. 
5. Pires BBC, Miranda JG, Freitas FA. O treinamento dos musclos do assoalho pélvico com adição do biofeedback na redução dos sintomas de incontinência urinaria em mulheres. Uma revisão de literatura. Revista Digital. 2013;17(176):1.

6. Bernards ATM, Berghmans BCM, Slieker-TenHove MCP, Staal JB, de Bie RA, Hendriks EJ. Dutch guidelines for physiotherapy in patients with stress urinary incontinence: an update. Int Urogynecol J. 2014; 25(2):171-179.

7. Oliveira AIA, Silva RLM, Zaparoli DA. Inovação tecnológica e inclusão social. Belém: EDUEPA, 2011.

8. Reading SA, Prickett K. Evaluation of children playing a newgeneration motion-sensitive active videogame by accelerometry and indirect calorimetry. Games Health J. 2013; 2(3):166-173

9. Deffieux X, Hubeaux K, Faivre E, Raibaut P, Ismael SS, Fernandez $\mathrm{H}$, et al. Sacral reflexes and urinary incontinence in women: new concepts. Ann Phys Rehabil Med 2009;52:256-68.

10. Dumoulin C, Cacciari LP, Hay-Smith EJC. Pelvic floor muscle training versus no treatment, or inactive control treatments, for urinary incontinence in women. Cochrane Database Syst Rev. 2018;10(10):CD005654. Published 2018 Oct 4.

11. Bø K, Berghmans B, Mørkved S, Van Kampen M. Evidence-Based Physical Therapy for the Pelvic Floor: Bridging Science and Clinical Practice. 2 ed. London: Churchill Livingstone, 2015, 446 p.

12. Nie XF, Ouyang $Y Q$, Wang L, Redding SR. A meta-analysis of pelvic floor muscle training for the treatment of urinary incontinence. Int J Gynaecol Obstet. 2017;138(3):250255.

13. García-Sánchez E, Ávila-Gandía V, López-Román J, Martínez-Rodríguez A, Rubio-Arias JÁ. What Pelvic Floor Muscle Training Load is Optimal in Minimizing Urine Loss in Women with Stress Urinary Incontinence? A Systematic Review and Meta-Analysis. Int J Environ Res Public Health. 2019;16(22):4358.
14. Berghmans B. Physiotherapy Assessment to Identify The '5 Fs': A Concept of Innovative Pelvic Floor Training. Int J Phys Med Rehabil. 2020; 8(4):1-3.

15. Berghmans B, Seleme MR, Bernards ATM. Physiotherapy assessment for female urinary incontinence. Int $\mathrm{J}$ Urogynecol 2020;31(5): 917-931.

16. Cruz AP, Lima TB. O uso da realidade virtual como ferramenta de inovação para reabilitação de pacientes com doença de Parkinson: uma revisão sistemática. Ciên Biol Saúde Unit. 2015; 2(3):97-110.

17. Andrade MF, Silva VR, Martinho N, Marques J, Carvalho $L$, Riccetto C, et al. A realidade virtual e a cinesioterapia como meios de treinamento no aumento da contratilidade dos músculos do assoalho pélvico e abdômen. In: Simpósio Internacional de Iniciação Cientifica e Tecnologia da USP, 220; 2014.

18. Bezerra LO, de Oliveira MCE, da Silva Filho EM, et al Impact of Pelvic Floor Muscles Training Isolated and Associated with Game Therapy on Mixed Urinary Incontinence: A Randomized Controlled Trial [published online ahead of print, 2020 Jul 21]. Games Health J. 2020;10.1089/ g4h.2019.0207.

19. Peixinho TAM, Saraiva A, Trippo KV. Efeitos da realidade virtual na função muscular em mulheres com incontinência urinária: relatos de caso. Rev Pesq Fisio. 2018; 8(3):387-396

20. Nascimento MS, Trippo KV, Saraiva A. Terapia por ex posição à realidade virtual nofortalecimento do assoalho pélvico: uma revisão sistemática. SFM. 2017; 5(1):47-60.

\section{CORRESPONDÊNCIA}

Érica Feio Carneiro Nunes

Universidade do Estado do Pará,

Curso de Fisioterapia. R. do Una, 156

CEP: 66050-540, Belém, PA, Brasil.

E-mail: ericarneiro@yahoo.com.br 